FERMILAB-Pub-87/19-E

7000.733

7000.649

\title{
Is There a Way to Measure the Deep-Inelastic Cross-Section Using Wide-Band Neutrino Beams?
}

\author{
R. Belusevic \\ University of Florida, Gainesville, Florida 32611 USA \\ and \\ D. Rein \\ III. Phys. Institut, RWTH Aachen \\ D-5100 Aachen, F.R. Germany
}

January 1987 


\title{
IS THERE A WAY TO MEASURE THE DEEP-INELASTIC CROSS-SECTION USING WIDE-BAND NEUTRINO BEAMS?
}

\author{
R. Belusevic ${ }^{\ddagger}$ \\ University of Florida, Gainesville, Florida 32611 \\ and \\ D. Rein \\ III. Phys. Institut, RWTH Aachen \\ D-5100 Aachen, F.R. Germany
}

\begin{abstract}
A method is described for extracting the value of the neutrino flux in a wide-band beam exposure based on computed differential cross-sections for resonance production and quasielastic scattering of neutrinos on nucleons. The resonance production cross-sections, computed by means of a relativistic quark model, are in excellent agreement with measurements of various neutrino-induced exclusive final states performed so far and also with data from inclusive deepinelastic neutrino interactions. The theoretical uncertainty inherent in the method is estimated to be about $10 \%$ and is roughly equal to the systematic error which plagues the measurements of the neutrino flux in present-day narrow-band beams. Before the first Tevatron narrow-band beam data becomes available in a few years time, if at all, this method provides the only reliable way of "measuring" the total neutrino cross-section with the existing wide-band beam data, thus extending the measured energy range by nearly a factor of two.
\end{abstract}


The idea of using the theoretical value of the quasielastic differential cross-section $d \sigma / d Q^{2}$ to deduce the incident anti-neutrino flux with hydrogen targets is a rather old one ${ }^{1}$ :

The (anti)neutrino differential cross-section $d \sigma / d Q^{2}$ reads

$$
\frac{d \sigma^{\nu, \nu}}{d Q^{2}}=\frac{M^{2} G^{2} \cos ^{2} \theta_{c}}{8 \pi E^{2}}\left[A\left(Q^{2}\right) \pm B\left(Q^{2}\right) \frac{s-\mu}{M^{2}}+C\left(Q^{2}\right) \frac{(s-u)^{2}}{M^{4}}\right]=f\left(F_{V,} F_{A}\right)
$$

where

$Q^{2}=2 E\left(E_{\mu}-P_{\mu} \cos \theta_{\mu \nu}\right), \quad(s-u)=4 E M-Q^{2}-m_{\mu}^{2}$

$\mathrm{A}, \mathrm{B}$, and $\mathrm{C}$ are known functions of $Q^{2}$ which contain the quasi-elastic vector and axialvector formfactors $\mathrm{F}_{V}\left(Q^{2}\right)$ and $\mathrm{F}_{A}\left(Q^{2}\right)$, respectively. While $\mathrm{F}_{V}\left(Q^{2}=0\right) \equiv g_{V}=1$ by virtue of CVC, the axialvector formfactor attains the value $\mathrm{g}_{A}=-1.25$ at $Q^{2}=0$. It follows then from equation (1) that

$$
\left.\frac{d \sigma^{e l}}{d Q^{2}}\right|_{Q^{2}=0} ^{\nu}=\left.\frac{d \sigma^{e l}}{d Q^{2}}\right|_{Q^{2}=0} ^{D}=\frac{G^{2} \cos ^{2} \theta_{c}}{2 \pi}\left(1+\left|\frac{g_{A}}{g_{V}}\right|^{2}\right)
$$

is a constant, i.e. independent of the incident (anti)neutrino energy $E$ and thus can be used to obtain the (anti)neutrino flux with a hydrogen or deuterium target, respectively. Moreover, at large energies the total quasi-elastic cross-section turns out to be energy independent, in accord with theoretical prediction, making it possible to extract the shape of the neutrino spectrum even off nuclear targets without explicit knowledge of the form factors and effects of Fermi motion and the Pauli exclusion principle. Unfortunately, this method relies on being able to identify quasi-elastic events, which is far from easy when using heavy nuclear targets.

A way out of this problem is to use deep inelastic neutrino data and a theoretical prediction of the values of the differential cross-sections for resonance production and quasi-elastic scattering of neutrinos on nucleons and to combine them in a reliable way. The point is that in a certain kinematical region the total inclusive cross-section will entirely be due to quasi-elastic and resonance production processes. Thus any inclusive cross section measurement in this kinematical domain is immediately linked to a few exclusive reactions whose cross-sections are theoretically 
calculable and fairly energy independent at high enough energies ( $E \gtrsim 10-20 \mathrm{GeV}$ ). Our method, which will be described in some detail, is based on the computation of the differential quasielastic and resonance cross-section expressed in the form $d \sigma / d y$, where $\mathrm{y}$ denotes the familiar scaling variable $y=\nu / E$. The quasi-elastic part $d \sigma^{e l} / d y=2 M E d \sigma^{e l} / d Q^{2}$ can be obtained in a straightforward manner. Recent quark model calculations of $d \sigma^{\text {res }} / d y=E \int d Q^{2} d \sigma^{\text {res }} / d Q^{2} d \nu$ have been shown to account well for resonance-dominated single pion production data accumulated sor far ${ }^{2}$. Therefore, we will particularly rely on them when considering the general inclusive neutrino cross-section $d \sigma / d y$ in more detail at $y$-values close to zero.

According to the parton picture of the deep inelastic neutrino-nucleon scattering, the differential cross-section $d \sigma / d y$ at high neutrino energies is generally flat. This is indicated qualitatively in Fig. 1. For $y \rightarrow 0$ a wiggly structure will arise from resonance production and quasi-elastic scattering which determines $d \sigma / d y$ at exactly $y=0$. Similar arguments apply to high energy antineutrino-nucleon reactions whose limiting value at $y=0$ is likewise fixed by equation (2). Let us discuss matters now in a more quantitative way.

The shape of the deep inelastic part of the cross-section is governed by the QCD evolution of the structure functions with momentum transfer $Q^{2}$ :

$$
\frac{d \sigma^{\nu, \nu}}{d y}=\frac{G^{2} \cos ^{2} \theta_{c} M E}{\pi} \int_{0}^{1} d x\left[(1-y) F_{2}\left(x, Q^{2}\right)+\frac{y^{2}}{2} 2 x F_{1}\left(x, Q^{2}\right) \pm y\left(1-\frac{y}{2}\right) x F_{3}\left(x, Q^{2}\right)\right]
$$

Taking this expression at $y=0$ and neglecting the slow $y$-variation implicit in the additional $Q^{2}$-dependence of the structure functions $\left(Q^{2}=2 M E x y\right)$ one finds

$$
\left.\frac{d \sigma}{d y}\right|_{y \rightarrow 0} ^{\nu}=\left.\frac{d \sigma}{d y}\right|_{y \rightarrow 0} ^{\nu} \sim E \int_{0}^{1} d x F_{2}\left(x, Q^{2}\right) \sim E \cdot \text { const }
$$

where charge symmetry was invoked in assuming $F_{2}^{\nu}=F_{2}^{\nu}$. Thus, for $y \rightarrow 0$, the deep inelastic cross-section (3) divided by $\mathrm{E}$ tends to become constant, i.e. independent of $\mathrm{E}$.

The resonance production part of the cross-section is well described by means of a relativistic quark model due to Feynman, Kislinger and Ravndal ${ }^{3}$ (FKR-model), which has been applied to neutrino reactions by Ravndal ${ }^{4}$ and by Rein and Sehgal ${ }^{2,5}$. The authors of ref. 2 included resonance decay into nucleon and pion. Overlapping resonances were allowed to interfere and some nonresonant background of isospin 1/2 was also included. This background, however, can be disposed of ${ }^{5}$ if the form factors of higher resonances are adjusted such as to reproduce the 
Fig. 1

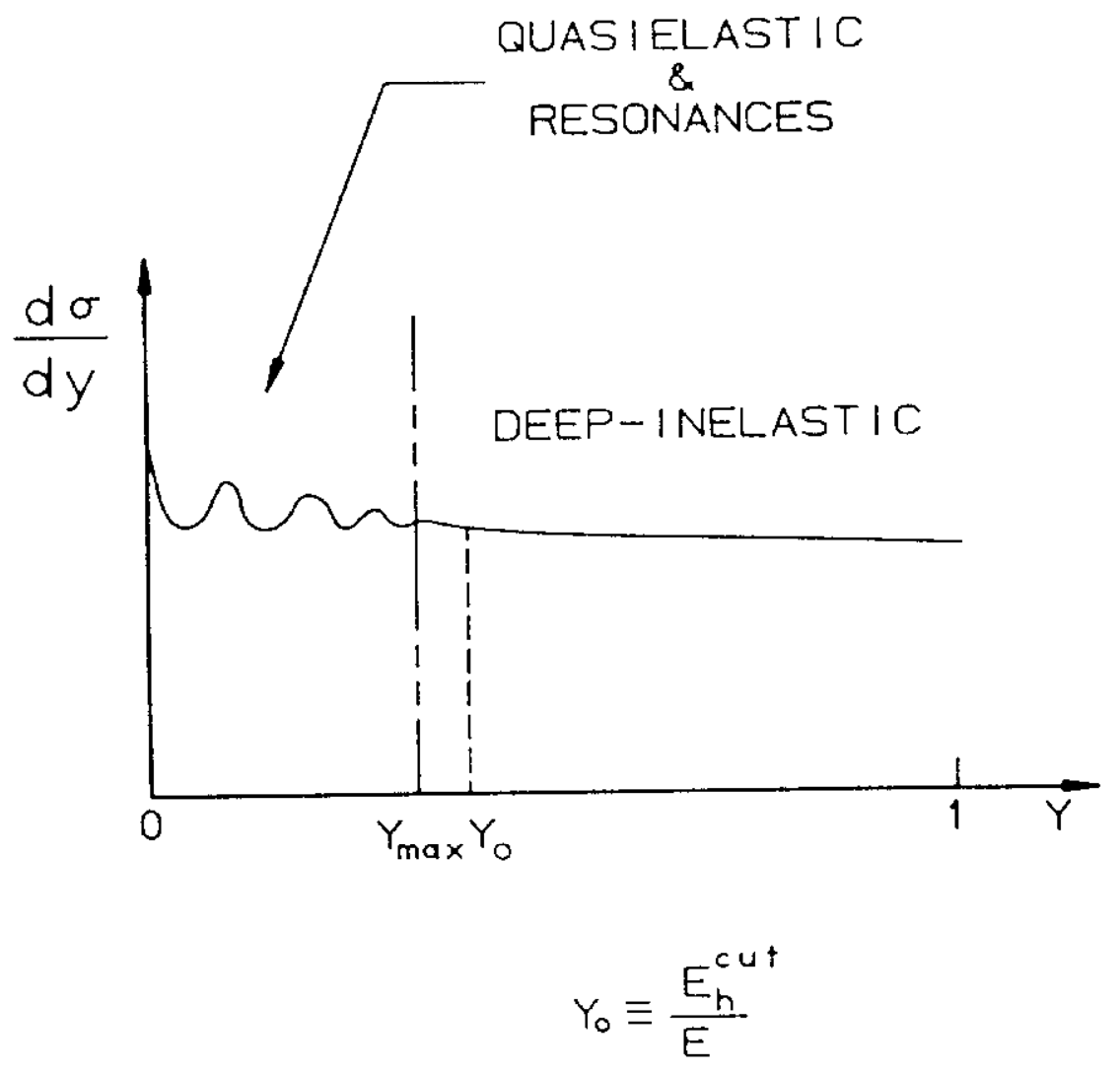


measured $Q^{2}$ - distribution. Figs. 2 through 4 show a selection of results from ref. 2 compared with data from neutrino-nucleon interactions. For invariant energy $\mathrm{W} \lesssim 1.4 \mathrm{GeV}$ essentially only one resonance, the $\Delta(1234)$, can contribute. Its predicted cross-section at $Q^{2}=0$ is numerically very close to

$$
\left.\frac{d \sigma}{d Q^{2}}\right|_{Q^{3}=0}=\frac{G^{2} \cos ^{2} \theta_{c}}{\pi^{2}} \frac{E^{\prime}}{E} \frac{f_{\pi}^{2}}{|\widetilde{Q}|} \sigma_{N \pi \rightarrow \Delta}
$$

as required by PCAC. Ultimately, the excellent agreement with data in Figs. 3 and 4 bears witness to the adequacy of this theoretical description.

Considering the kinematical domain of $y$

$$
y=\frac{\nu}{E}=\frac{W^{2}-M^{2}+Q^{2}}{2 M E}
$$

where quasi-elastic scattering and resonance production build up the total inclusive cross-section, one obtains the following limits:

$$
0=y_{\min } \leq y \leq y_{\max }=\frac{W_{\max }^{2}-M^{2}}{2 M E}
$$

Above $\mathrm{W}=2 \mathrm{GeV}$ resonances become less important. Therefore, it seems reasonable to fix $\mathrm{W}_{\max }$ at $2 \mathrm{GeV}$, as has been done in ref. 2. Numerically then

$$
y_{\max }=\frac{4-0.88}{1.88} \frac{\mathrm{GeV}}{E} \simeq 1.7 \frac{\mathrm{GeV}}{E}
$$

with $\mathrm{E}$ measured in GeV. Obviously $y_{\max } \sim 1 / E$ and correspondingly the y-interval $\Delta y=$ $y_{\max }-y_{\min }$ shrinks with increasing neutrino energy. On the other hand, the differential crosssection $d \sigma / d y$ for resonance production and quasi-elastic scattering increases linearly with $\mathrm{E}$, thus keeping the total cross-section energy independent.

Evaluation of $\sigma$ for proton and neutron targets then leads to

$$
\left.\begin{array}{l}
\sigma^{e l}(n)=0.74 \cdot 10^{-38} \mathrm{~cm}^{2} \\
\sigma^{e l}(p)=0
\end{array}\right\} \sigma^{e l}(N)=0.37 .10^{-38} \mathrm{~cm}^{2}
$$


Fig. 2
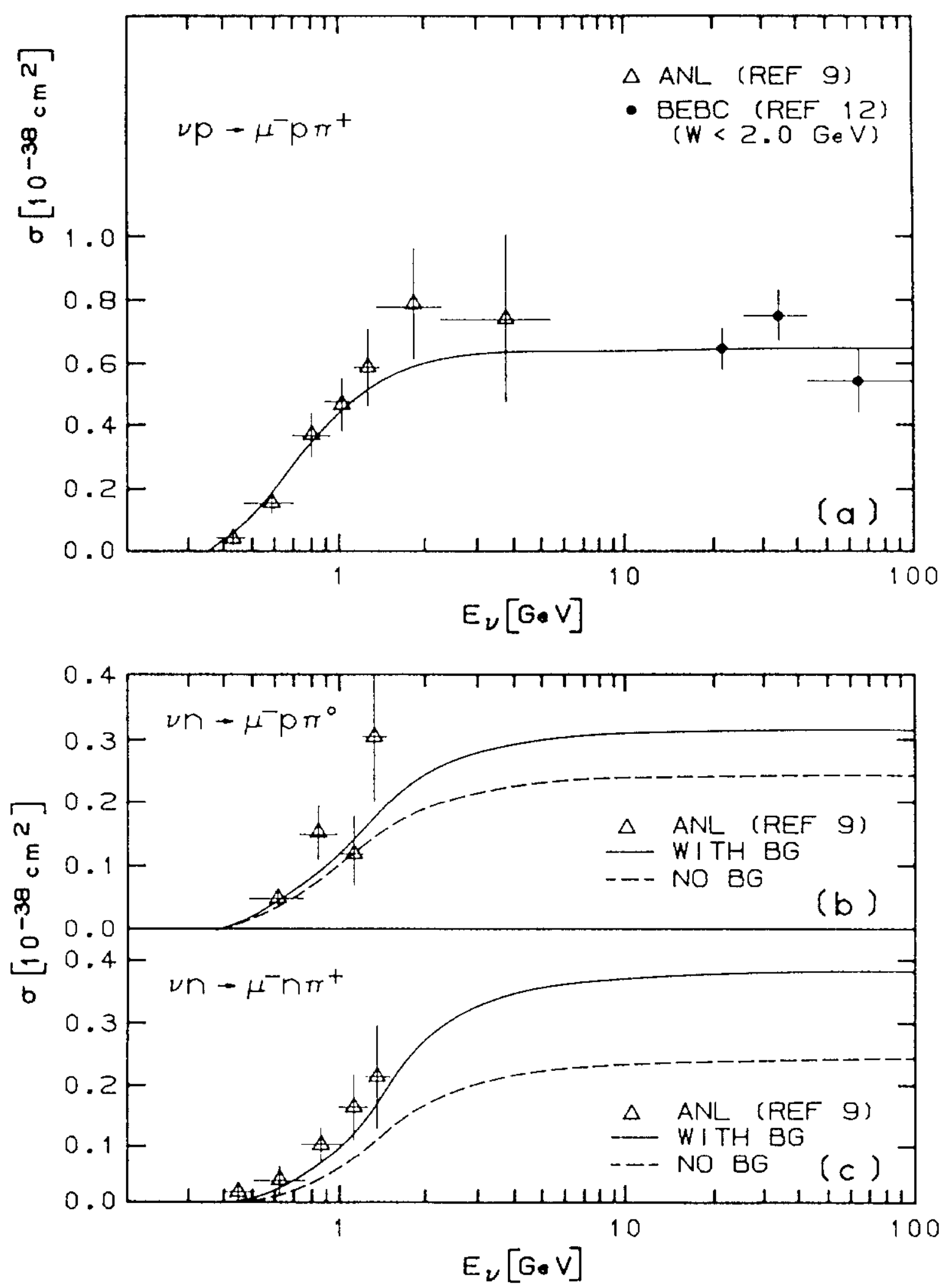
Fig. 3
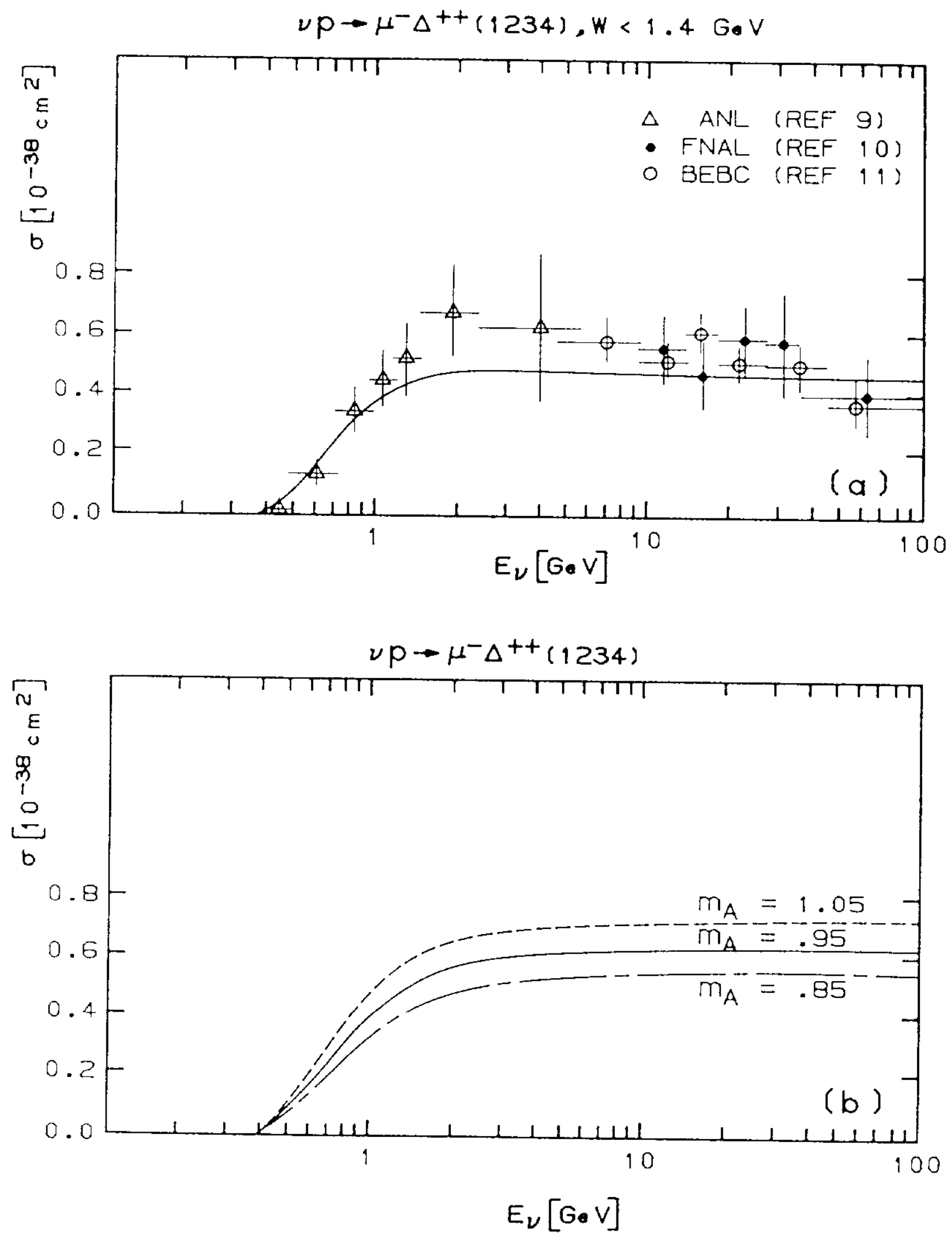
Fig. 4

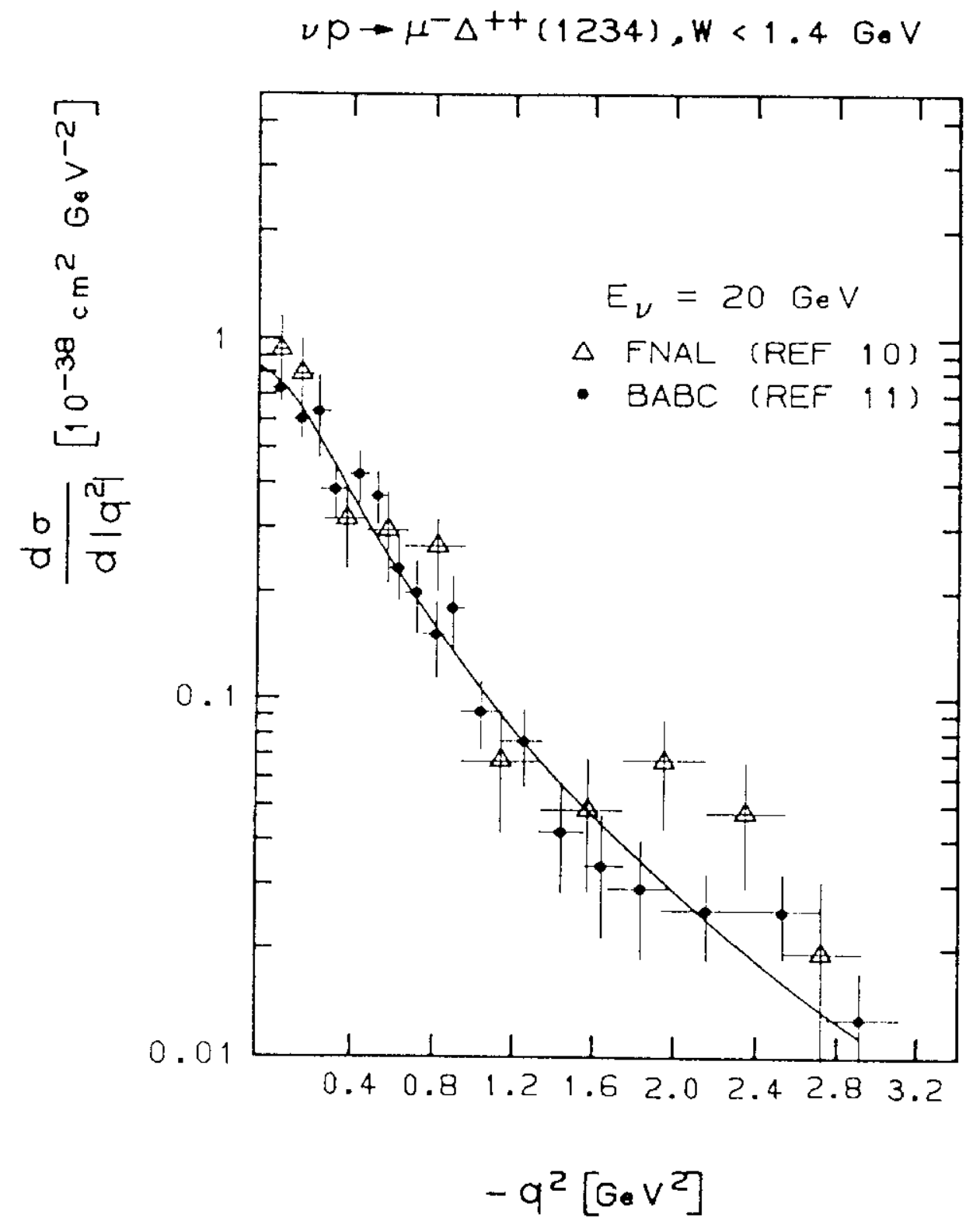

8 
and

$$
\left.\begin{array}{l}
\sigma^{r e s}(n)=0.75 \cdot 10^{-38} \mathrm{~cm}^{2} \\
\sigma^{r e s}(p)=0.74 \cdot 10^{-38} \mathrm{~cm}^{2}
\end{array}\right\} \sigma^{\text {res }}(N)=0.745 \cdot 10^{-38} \mathrm{~cm}^{2}
$$

independent of energy at high energies. In arriving at (8) and (9) the form factor mass parameters $m_{V}, m_{A}$ have been chosen ${ }^{6}$ as $m_{V}=.84 \mathrm{GeV} / \mathrm{c}^{2}$ and $m_{A}=1.0 \mathrm{GeV} / \mathrm{c}^{2}$. The calculation has been performed according to the procedure described in ref. 5 , which differs very little from the earlier one used in ref. 2. The resonance cross-sections (9) are somewhat smaller than the ones given in Table IV of ref. 2, because the restriction $y \leq y_{\max }$ cuts off contributions from the higher $Q^{2}$-domain. Averaging over proton and neutron targets and adding up the quasi-elastic and resonance contribution gives

$$
\sigma_{\text {tot }}(N)=1.12 \cdot 10^{-38} \mathrm{~cm}^{2} \quad\left(N=\frac{p+n}{2}\right)
$$

which may be written as

$$
\sigma_{\text {tot }}=\int d y\left(\frac{d \sigma}{d y}\right) \equiv\left(\frac{\overline{d \sigma}}{d y}\right) \Delta y
$$

resulting in

$$
\frac{1}{E}\left(\frac{\overline{d \sigma}}{d y}\right)=\frac{\sigma_{t o t}}{\Delta y}=0.68 \cdot 10^{-38} \mathrm{~cm}^{2} \mathrm{GeV}^{-1}
$$

This agrees quite well with the value of

$$
\left.\frac{1}{E} \frac{d \sigma}{d y}\right|_{y=0}=(0.719 \pm 0.035) \cdot 10^{-38} \mathrm{~cm}^{2} \mathrm{GeV}^{-1}
$$

obtained $^{7}$ by extrapolating a fit to deep inelastic data to $y=0$. Since $(1 / E)(d \sigma / d y)^{\text {inel }}$ is rather independent of $y$ in the energy range covered by the data $(40 \leq \mathrm{GeV}<E \leq 200 \mathrm{GeV})$, such an extrapolation is almost equivalent to measuring the value of $d \sigma / d y$ at the lowest $y$ value attainable in an actual experiment ${ }^{1}$.

Having found a good agreement between the theoretical prediction for an average differential cross-section in the vicinity of $y=0$ and of the extrapolated measured differential cross-section,

1 The cross-section extrapolated to $y=0$, however, has nothing to do with the cross-section value at exactly $y=0$, which is determined by the quasi-elastic part of the cross-section, as mentioned before. 
Fig. 5

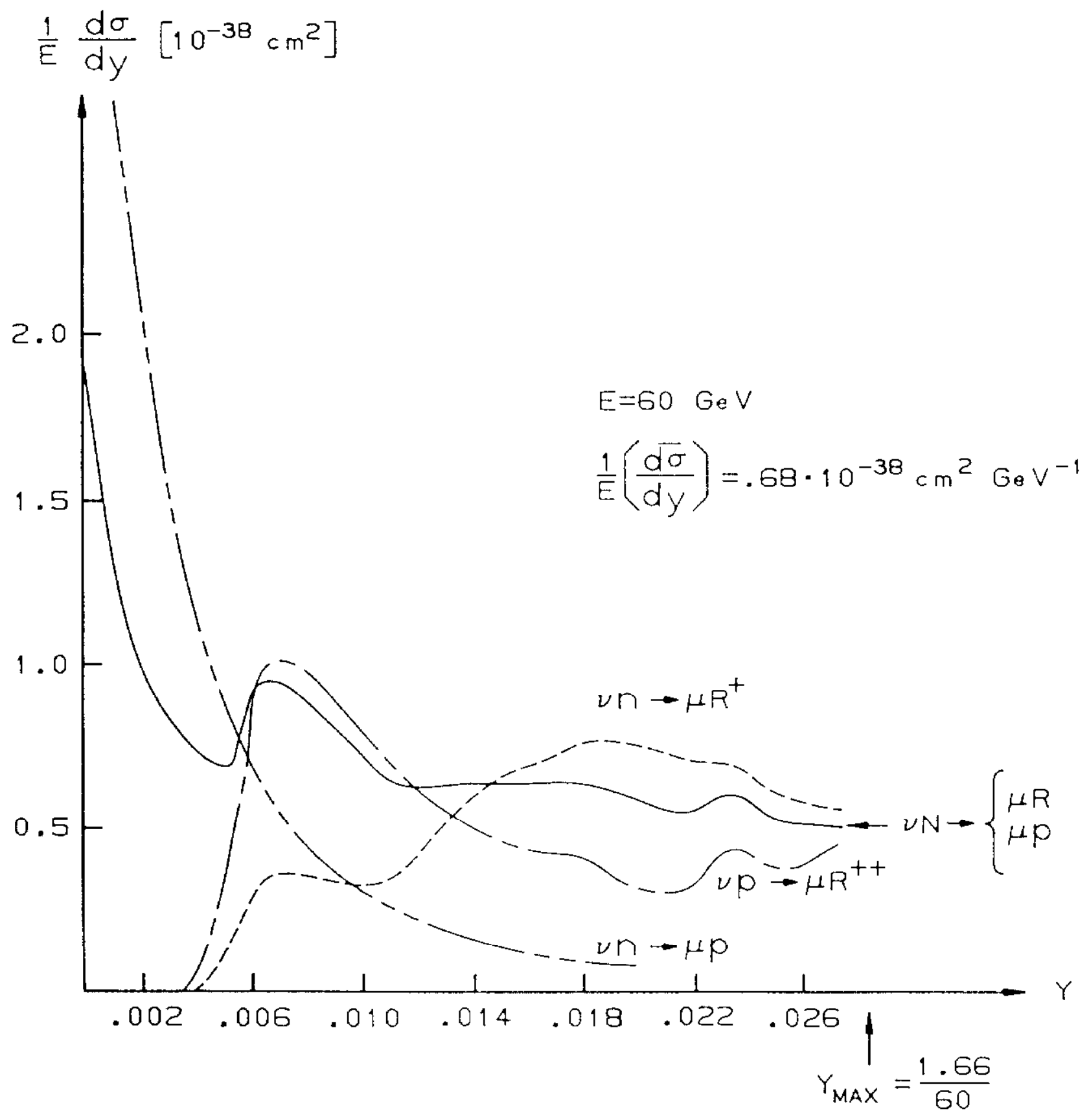


one is encouraged to use the above results for deducing the neutrino flux in a deep inelastic scattering experiment by going to very low $y$-values. If the lowest measured value of $y, y_{0}$, equals the end point $y_{\max }$ of the domain where $d \sigma / d y$ can be calculated with some confidence, one can immediately write

$$
n\left(y_{o}\right)=\left.\frac{d \sigma}{d y}\right|_{y=y_{o}} \cdot \phi(E)=\left.\frac{d \sigma}{d y}\right|_{y=y_{\max }} \cdot \phi(E)
$$

and obtain the value of the flux, $\phi(\mathbb{E})$, by dividing the number of measured events at $y_{o}, n\left(y_{o}\right)$, by the value of $d \sigma / d y \mid y=y_{o}$. The number of events at $y_{o}$ is obtained by fitting the corrected (for acceptance, etc.) data between $y_{o} \equiv E_{h}^{\text {cut }} / E \leq y \leq 1$, where $E_{h}^{\text {cut }}$ depends on the experiment in question and is in the range of $5-10 \mathrm{GeV}$ for present-day counter neutrino experiments.

A difficulty arises when joining the cross-section predicted up to $y_{\max }=1.7 \mathrm{GeV} / \mathrm{E}$ (see Fig. 5) with the $y$-distribution measured from $y_{o}=5 \mathrm{GeV} / \mathrm{E}$ onward: this experimental constraint makes $y_{o}$ fall outside the resonance-dominated $y$-interval. Hence, one is forced to make an assumption about the behaviour of the differential cross-section $d \sigma / d y$ between $y_{0}$ and $y_{\max }$, in order to be able to combine the computed cross-section with data. A guidance is provided by the observation of Bloom and Gilman ${ }^{8}$ that

“... the prominent resonances do not disappear at large $Q^{2}$ relative to a background under them, but instead (...) follow the scaling limit curve."

which is depicted in Fig. 6a, b below and is based on the measurements of the structure function $\nu W_{2}$ in inelastic electron-nucleon scattering. This observation, combined with the fact that the deep inelastic differential cross-section $d \sigma / d y$ is rather flat in $y$, as mentioned above, and with the results of the computation presented in Fig. 5 (showing that there is little change in the value of $d \sigma / d y$ as $y \rightarrow y_{\max }$ ), allows one to assume that $d \sigma /\left.d y\right|_{y=y_{0}} \simeq d \sigma /\left.d y\right|_{y=y_{\max }}$. More generally, one may assume a smooth connection between the very low $y$ domain, where $d \sigma / d y$ is explicitly calculable, and the region of larger $y$-values, where $d \sigma / d y$ is measured at a fixed $\mathrm{E}$, apart from normalization which requires $\phi(E)$.

The dominant uncertainties presented in this procedure are the following: First, there is a theoretical error from the quark model calculation of resonance production. This uncertainty is not very large (estimated to be about $10 \%$ ) and could probably be diminished by adjusting the 
Fig. Ga

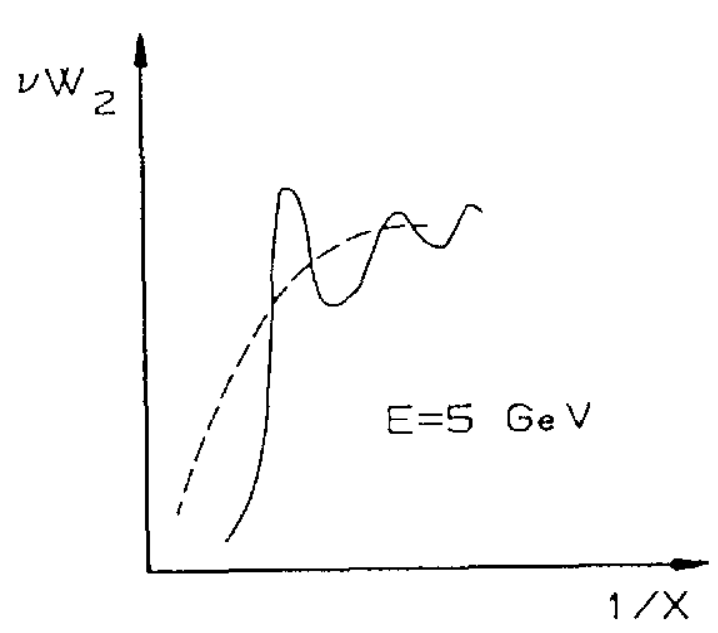

Flg. $6 b$

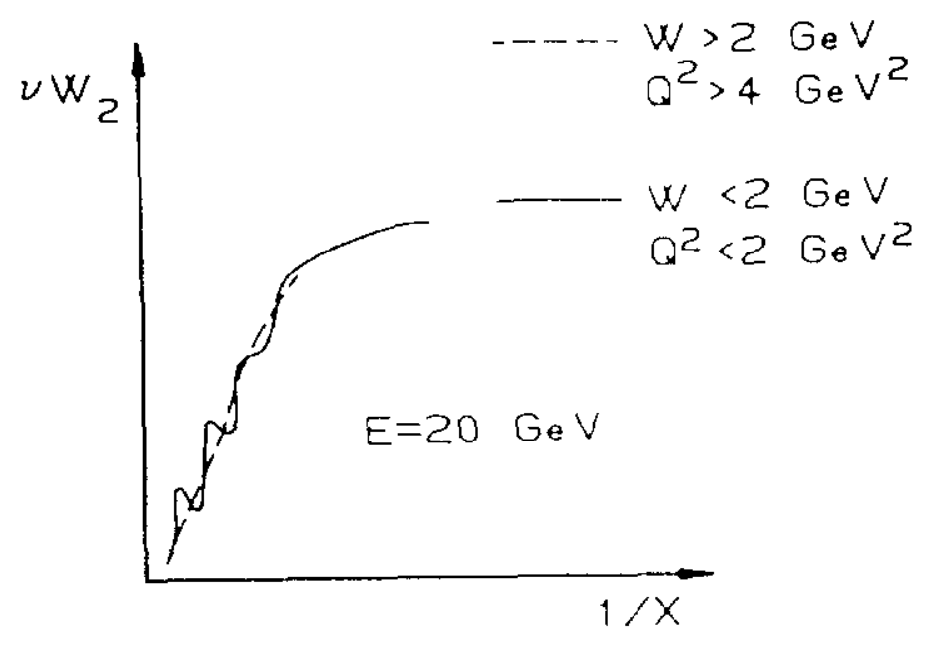


parameters of the model (mainly $m_{A}$ ) in an optimal way using the most accurate high energy pion production data which have little experimental uncertainty. The accuracy of the resonance model is, of course, best for low lying resonances which have large branching ratios into $\mathrm{N} \pi$. Close to the upper bound $W_{\max }=2 \mathrm{GeV}$ resonances from beyond $W=2 \mathrm{GeV}$ may leak in to some extent, although their small $\pi \mathrm{N}$-branching ratio may prevent them from being recorded in single pion measurements. This is an additional uncertainty which is difficult to estimate. Its impact, however, diminishes with decreasing $y$. Secondly, there is an error with which the value of $d \sigma / d y$ at $y_{o}$ is extracted from data and which is due to limited statistics. The main uncertainty lies in the extrapolation of $d \sigma / d y$ between $y_{\max }=1.7 \mathrm{GeV} / \mathrm{E}$ and $y_{o}=5 \mathrm{GeV} / \mathrm{E}$, which is minimized by going to high energies where $y_{o}-y_{\max } \sim 3.5 \mathrm{GeV} / \mathrm{E}$ becomes a small fraction of the total available $y$-interval.

The method described in this note can also be used to cross-check the value of the neutrino flux measured in narrow-band beams. Conversely, a precise measurement of the neutrino flux in a narrow-band beam exposure can be used to test its accuracy by comparing the values of the measured and computed fluxes, before applying the method to "measure" the neutrino flux in a wide band beam at higher energies.

\section{Acknowledgements}

It is a pleasure to thank L. M. Sehgal for his interest and advice. One of us gratefully acknowledges support from the German Bundesministerium für Forschung und Technologie.

†Work supported by the U.S. Department of Energy

\section{References}

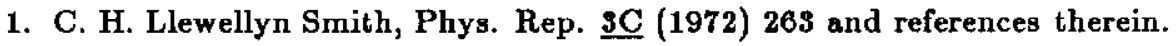

2. D. Rein and L. M. Sehgal, Ann. Phys. (N.Y.) 193 (1981) 79.

3. R. P. Feynman, M. Kislinger, andd F. Ravndal, Phys. Rev. D3 (1971) 2706.

4. F. Ravndal, Nuovo Cim. $\underline{A 18}$ (1973) 385; Lett. N.C. $\underline{3}$ (1972) 631.

5. D. Rein, to be published in Z. Phys. C. 
6. S. V. Belikov et al., Z. Phys. A320 (1985) 625.

7. F. Sciulli, in: Proc. 1977 Intl. Sympos. on Lepton and Photon Interactions at High Energies, Ed. F. Gutbrod, Hamburg 1977, p. 239.

8. E. Bloom and F. Gilman, Phys. Rev. Let. $\underline{25}$ (1970) 1140.

9. S. J. Barish et al., Phys. Rev. D19 (1979) 2521, J. Campbell et al., Phys. Rev. Lett. $\underline{30}$ (1973) 335.

10. J. Bell et al., Phys. Rev. Lett. 41 (1978) 1008, 1012.

11. P. Allen et al., Nucl. Phys. B176 (1980) 269.

12. P. Allen, BEBC Collab.; private communication with D. Rein. 